\section{TAK1 is a pivotal therapeutic target for tumor progression and bone destruction in myeloma}

\author{
Jumpei Teramachi, ${ }^{1,2}$ Hirofumi Tenshin,, ${ }^{2,3}$ Masahiro Hiasa, ${ }^{2,3}$ Asuka Oda, ${ }^{2}$ \\ Ariunzaya Bat-Erdene, ${ }^{2,4}$ Takeshi Harada, ${ }^{2}$ Shingen Nakamura, ${ }^{2}$ \\ Mohannad Ashtar, ${ }^{2,3}$ So Shimizu, ${ }^{2,3}$ Masami Iwasa, ${ }^{2}$ Kimiko Sogabe, ${ }^{2}$ \\ Masahiro Oura, ${ }^{2}$ Shiro Fujii, ${ }^{2}$ Kumiko Kagawa, ${ }^{2}$ Hirokazu Miki, ${ }^{5}$ Itsuro Endo, ${ }^{6}$ \\ Tatsuji Haneji, ${ }^{1}$ Toshio Matsumoto ${ }^{7}$ and Masahiro Abe $^{2}$ \\ ${ }^{1}$ Department of Histology and Oral Histology, Tokushima University Graduate School, \\ Tokushima, Japan; '2Department of Hematology, Endocrinology and Metabolism, \\ Tokushima University Graduate School, Tokushima, Japan; ${ }^{3}$ Department of Orthodontics \\ and Dentofacial Orthopedics, Tokushima University Graduate School, Tokushima, Japan; \\ ${ }^{4}$ Department of Immunology and Laboratory Medicines, School of Biomedicine, \\ Mongolian National University of Medical Sciences, Ulaanbaatar, Mongolia; ${ }^{5}$ Division of \\ Transfusion Medicine and Cell Therapy, Tokushima University Hospital, Tokushima, \\ Japan; ${ }^{6}$ Department of Chronomedicine, Institute of Biomedical Sciences, Tokushima \\ University Graduate School, Tokushima, Japan and ${ }^{7}$ Fujii Memorial Institute of Medical \\ Sciences, Tokushima University, Tokushima, Japan
}

\section{ABSTRACT}

A long with tumor progression, the bone marrow microenvironment is skewed in multiple myeloma (MM), which underlies the unique pathophysiology of MM and confers aggressiveness and drug resistance in $\mathrm{MM}$ cells. TGF- $\beta$-activated kinase-1 (TAK1) mediates a wide range of intracellular signaling pathways. We demonstrate here that TAK1 is constitutively overexpressed and phosphorylated in MM cells, and that TAK1 inhibition suppresses the activation of NF- $\kappa B$, p38MAPK, ERK and STAT3 in order to decrease the expression of critical mediators for MM growth and survival, including PIM2, MYC, Mcl1, IRF4, and Sp1, along with a substantial reduction in the angiogenic factor VEGF in MM cells. Intriguingly, TAK1 phosphorylation was also induced along with upregulation of vascular cell adhesion molecule-1 (VCAM-1) in bone marrow stromal cells (BMSC) in cocultures with MM cells, which facilitated MM cell-BMSC adhesion while inducing IL- 6 production and receptor activator of nuclear factor $\kappa-B$ ligand (RANKL) expression by BMSC. TAK1 inhibition effectively impaired MM cell adhesion to BMSC to disrupt the support of MM cell growth and survival by BMSC. Furthermore, TAK1 inhibition suppressed osteoclastogenesis enhanced by RANKL in cocultures of bone marrow cells with MM cells, and restored osteoblastic differentiation suppressed by MM cells or inhibitory factors for osteoblastogenesis overproduced in MM. Finally, treatment with the TAK1 inhibitor LLZ1640-2 markedly suppressed MM tumor growth and prevented bone destruction and loss in mouse MM models. Therefore, TAK1 inhibition may be a promising therapeutic option targeting not only MM cells but also the skewed bone marrow microenvironment in MM.

\section{Introduction}

Multiple myeloma (MM) has a unique propensity to almost exclusively develop in the bone marrow and generate devastating bone destruction. MM cells enhance osteoclast $(\mathrm{OC})$ formation and activity, and suppress osteoblastic differentiation from bone marrow stromal cells (BMSC), leading to extensive bone destruction with rapid development of osteolytic lesions. ${ }^{1,2}$ Angiogenesis is also enhanced through these cellular interactions. ${ }^{3,4}$ The types of cells surrounding MM cells create a cellular microenvironment suitable for MM cell growth and survival to confer drug resistance, which can be termed the "MM niche".
Ferrata Storti Foundation

Haematologica 2021

Volume 106(5):1401-1413

\section{Correspondence:}

JUMPEI TERAMACHI

jumptera@okayama-u.ac.jp

MASAHIRO ABE

masabe@tokushima-u.ac.jp

Received: August 7, 2019.

Accepted: April 2, 2020.

Pre-published: April 9, 2020.

https://doi.org/10.3324/haematol.2019.234476

(C)2021 Ferrata Storti Foundation

Material published in Haematologica is covered by copyright. All rights are reserved to the Ferrata Storti Foundation. Use of published material is allowed under the following terms and conditions:

https://creativecommons.org/licenses/by-nc/4.0/legalcode. Copies of published material are allowed for personal or internal use. Sharing published material for non-commercial purposes is subject to the following conditions:

https://creativecommons.org/licenses/by-nc/4.0/legalcode, sect. 3. Reproducing and sharing published material for commercial purposes is not allowed without permission in writing from the publisher. 
In order to improve therapeutic efficacy, we need to disrupt the MM niche that confers drug resistance. Therefore, we looked for novel molecules upregulated in the $\mathrm{MM}$ niche to be targeted, and found that proviral integrations of Moloney virus 2 kinase (PIM2) is constitutively overexpressed as an anti-apoptotic mediator in MM cells. ${ }^{5}$ PIM2 expression has been demonstrated to be higher in hematologic malignancies than solid cancers or their normal tissue counterparts, and highest in $\mathrm{MM}^{6,7}$ Importantly, PIM2 can be further upregulated in MM cells in cocultures with BMSC as well as OC. ${ }^{5}$

Although multiple soluble inhibitors for osteoblastogenesis have been reported to be overproduced in $\mathrm{MM}$, including IL-3, ${ }^{8}$ IL-7, ${ }^{9}$ TNF- $\alpha,{ }^{10}$ TGF- $\beta,{ }^{11}$ and activin $A,{ }^{12}$ PIM2 was found to be upregulated in BMSC by these inhibitory factors acting as a common intracellular mediator to suppress their osteoblastogenesis. ${ }^{13}$ Moreover, we subsequently reported that PIM2 is induced in osteoclastic lineage cells by receptor activator of nuclear factor $\kappa-B$ ligand (RANKL) to act as a critical mediator of RANKLinduced osteoclastogenesis in $\mathrm{MM} .{ }^{14}$ Therefore, PIM2 appears to play a versatile role in tumor progression and bone destruction and bone loss in $\mathrm{MM}$, and is regarded as an important therapeutic target.

TGF- $\beta$-activated kinase 1 (TAK1) is a member of the mitogen-activated protein kinase kinase (MAP3K) family, also known as MAP3K7..$^{15,16}$ It was originally identified as a key kinase in transducing TGF- $\beta$ signaling down to p38 mitogen-activated protein kinase (MAPK) and c-Jun and $\mathrm{N}$-terminal kinase (JNK). ${ }^{15}$ Subsequently, TAK1 has been demonstrated to be associated with the activation of a wide range of intracellular signaling pathways important for various cellular functions, including the activation of nuclear factor- $\kappa \mathrm{B}(\mathrm{NF}-\kappa \mathrm{B})$ and extracellular signal-regulated kinase (ERK). ${ }^{15}$ Therefore, TAK1 appears to be a gate keeper to facilitate the multiple important intracellular signaling pathways. Therapeutic efficacy of TAK1 inhibition has been preclinically demonstrated in different types of cancers, including mantle cell lymphoma, ${ }^{17}$ breast cancer, ${ }^{18}$ and colon cancer. ${ }^{19}$

We demonstrate here that TAK1 is constitutively overexpressed and phosphorylated in MM cells and that TAK1 acts as an upstream regulator responsible for multiple signaling pathways critical for $\mathrm{MM}$ growth and survival. ${ }^{1}$ TAK1 phosphorylation is also induced in BMSC in cocultures with MM cells, which facilitates $M M$ cell adhesion to BMSC between very late antigen-4 (VLA-4) and vascular cell adhesion molecule-1 (VCAM-1), thereby inducing IL-6 production and RANKL expression by BMSC. Importantly, TAK1 inhibition was able to effectively induce MM cell death, and alleviate bone destruction though suppression of osteoclastogenesis and restoration of osteoblastogenesis. Therefore, TAK1 appears to be a pivotal therapeutic target in MM to disrupt the key signal transduction pathways responsible for tumor progression and bone destruction.

\section{Methods}

\section{Ethics}

All procedures involving human samples from healthy donors and patients were performed with written informed consent in accordance with the Declaration of Helsinki and a protocol approved by the Institutional Review Board for human protec- tion at the University of Tokushima (Permission number: 240). All animal experiments were conducted under the regulation and permission of the Animal Care and Use Committee of Tokushima University, Tokushima, Japan (toku-dobutsu 13094).

\section{Reagents}

Reagents used in this manuscript are described in the Online Supplementary Appendix.

\section{Cells and cell culture}

Details of the cells and cell culture procedures are available in the Online Supplementary Appendix.

\section{Western blotting}

Cells were collected and lysed in RIPA lysis buffer (Santa Cruz, Dallas, TX, USA). For cytosolic and nuclear preparation, cells were lysed in NE-PER extraction reagent (Thermo Fisher Scientific, Waltham, MA) in accordance with the manufacturer's protocol. Western blot analysis was done with equal protein amounts of cell lysate, as described previously. ${ }^{13}$

\section{Cell viability}

Cell viability was determined using the Cell Counting Kit-8 assay (Dojindo, Kumamoto, Japan) in accordance with the manufacturer's instructions. The absorbance of each well was measured at 450-655 nm using an iMark microplate reader (Bio-Rad Laboratories, Hercules, CA, USA). In order to assess apoptotic cells, cells were stained with an annexinV-FITC and propidium iodide labeling kit (MEBCYTO Apoptosis Kit; MBL, Nagano, Japan) in accordance with the manufacturer's instruction, and analyzed by flow cytometry.

\section{Small interfering RNA transfection}

Small interfering RNA (siRNA) transduction was performed as described previously. ${ }^{5,20}$ Human and mouse TAK1 siRNA were purchased from Santa Cruz. Human TAK1 siRNA was transfected into MM cells using a Human Nucleofector Kit (Lonza, Basel, Switzerland). Mouse TAK1 siRNA was transfected into mouse BMSC or RAW264.7 cells using siRNA Transfection Reagent (Santa Cruz) in accordance with the manufacturer's protocol.

\section{Real-time reverse transcription polymerase chain reaction}

RNA isolation and quantitative real-time reverse transcription polymerase chain reaction (RT-PCR) were performed as described previously. ${ }^{20}$ The following primer sequences were used: human RANKL F: TCGTTGGATCACAGCACATCA and R: TATGGGAACCAGATGGGATGTC, human IL-6 F: TCTGAGGCTCATTCTGCCCTCGAGC and R: AACTGGACCGAAGGCGCTTGTGGA, human GAPDH, used as an endogenous control to normalize each sample, F: TGTCTTCACCACCATGGAGAAGG and R: GTGGATGCAGGGATGATGTTCTG

\section{Adhesion assays}

Adhesion assays were performed as described previously. ${ }^{21}$ Human BMSC $\left(2 \times 10^{4}\right.$ cells/well) were expanded in 96-well culture plates. MM cells were labeled with BCECF-AM (Dojindo) for 2 hours at $37^{\circ} \mathrm{C}$ and $5 \% \mathrm{CO}_{2}$ as described previously. BMSC were washed, and the fluorescence-labeled MM cells were added onto the BMSC and incubated for 4 hours. Nonadherent BCECF-AM-labeled cells were removed by gently pipetting four times. Adherent cells were quantitated in a fluorescence multi-well plate reader (Infinite ${ }^{\circledR} 200$ PRO, TECAN, Männedorf. Switzerland). 


\section{Multiple myeloma animal model and histological analyses}

Multiple myeloma (MM) mouse models were prepared by intra-tibial inoculation of mouse luciferase-transfected 5TGM1 MM cells (a gift from Dr. Gregory R. Mundy [Vanderbilt Center for Bone Biology, Vanderbilt University, Nashville, TN, USA]) into ICR nu/nu mice (CLEA Japan) at 4-6 weeks old as described previously. ${ }^{13,14}$ The assessment of tumor growth and bone volume, and bone histomorphometric and immunohistochemical analyses are described in the Online Supplementary Appendix.

\section{Statistical analysis}

Statistical analysis was performed using Student's $t$-test or oneway analysis of variance (ANOVA). $P<0.05$ was considered as a significant difference. All statistics were performed using the Statistical Package for Social Sciences (SPSS 13.0 for Windows; Chicago, IL, USA).

\section{Results}

\section{TAK1 is activated to mediate growth and survival of multiple myeloma cells}

We first examined the expression and activation status of TAK1 in MM cells. TAK1 is highly overexpressed and phosphorylated in MM cell lines and primary MM cells from patients, whereas normal peripheral blood mononuclear cells (PMBC) only weakly expressed TAK1 (Figure 1A). IRF4 and MYC have been regarded as master regulators for MM cell survival and function, which MM cells are addicted to, and their reduction is a major mechanism for the anti-MM activity of immunomodulatory drugs lenalidomide and pomalidomide. ${ }^{23,24}$ Notably, the TAK1 inhibitor LLZ dose-dependently reduced MYC and IRF4 in parallel with the reduction in PIM2 expression and the phosphorylation of the PIM2 substrate 4E-BP1 as well as the anti-apoptotic factor Mcl-1 in MM cells (Figure 1B, left). We and others reported that transcription factor Sp1 is constitutively overexpressed in MM cells, which can serve as an important therapeutic target for MM. ${ }^{25-27}$ TAK1 inhibition also substantially reduced $\mathrm{Sp} 1$ (Figure 1B, left). The effects of TAK1 inhibition were confirmed with TAK1 knockdown using siRNA (Figure 1B, right).

TAK1 inhibition with LLZ dose-dependently induced cell death in all MM cell lines tested (Figure. 1C, left), and TAK1 knockdown also reduced the viability of MM cells (Figure 1C, right). The induction of apoptosis was confirmed using annexin $V$ and propidium iodide dual staining (Figure 1D) with activation of caspase-8, caspase-9, and caspase-3 (Figure 1E) in MM cells, indicating activation of the extrinsic as well as the intrinsic caspase-mediated apoptotic pathways. LLZ at the concentrations up to $10 \mu \mathrm{M}$ did not apparently impair the viability of normal PBMC (Online Supplementary Figure S1). Therefore, TAK1 appears to be a good therapeutic target to effectively induce cell death in $\mathrm{MM}$ cells through suppression of PIM2 plus other pro-survival mediators.

\section{TAK1 inhibition is able to abolish IL-6 and TNF- $\alpha$-induced signaling in multiple myeloma cells}

IL- 6 and TNF- $\alpha$ are predominant paracrine factors overproduced in the bone marrow microenvironment in $\mathrm{MM}$, and these elicit the signaling pathways responsible for MM cell growth and survival. ${ }^{28}$ As we demonstrated previously, ${ }^{5}$ PIM2 was substantially upregulated in MM cell lines in cocultures with BMSC as well as in the presence of IL-6 or TNF- $\alpha$ (Figure 2A). However, treatment with LLZ was able to abolish PIM2 upregulation in MM cells by BMSC as well as IL- 6 or TNF- $\alpha$. We further examined the effects of TAK1 inhibition on the signaling pathways in MM cells activated by IL- 6 and TNF- $\alpha$. After starving without serum, phosphorylation of TAK1 was reduced in RPMI8226 cells, but IL- 6 (Figure 2B) and TNF- $\alpha$ (Figure 2C) promptly induced the phosphorylation of TAK1 and activation of their corresponding downstream signaling molecules in serum-depleted media. Treatment with LLZ abolished IL-6-induced phosphorylation of STAT3 (Figure $2 \mathrm{~B})$, and TNF- $\alpha$-induced phosphorylation and degradation of $\mathrm{I} \kappa \mathrm{B} \alpha$ and phosphorylation of p38MAPK and ERK (Figure 2C) in the MM cells. Analyses of nuclear extracts from MM cells treated with TNF- $\alpha$ showed the nuclear accumulation of p65; however, treatment with LLZ reduced the $\mathrm{p} 65$ content in the nuclear extracts in the presence of TNF- $\alpha$ (Figure 2D). Consistent with the notion that PIM2 is transcriptionally upregulated in MM cells by the NF- $\kappa$ B signaling pathway, ${ }^{5}$ the PIM inhibitor SMI-16a showed only marginal effects on the nuclear accumulation of p65 induced by TNF- $\alpha$ (Figure 2D). Of note, the TAK1 inhibitor LLZ as well reduced MM cell viability in cocultures with BMSC (Figure 2E). TAK1 knockdown in MM cells mostly reduced their viability even in cocultures with BMSC (Figure 2F). Also, TAK1 knockdown in BMSC partially but significantly reduced their supportive activity for MM cell growth (Figure 2G). Therefore, TAK1 activation in both MM cells and BMSC is suggested to play an important role in $\mathrm{MM}$ cell growth and survival in cocultures with BMSC. Furthermore, although cytotoxic effects of doxorubicin on MM cells were blunted in cocultures with BMSC, TAK1 inhibition with LLZ was able to resume MM cell death by doxorubicin even in the presence of BMSC (Online Supplementary Figure S2). These results suggested that TAK1 inhibition impairs MM cell growth and survival supported by the bone marrow microenvironment.

\section{TAK1 inhibition impairs multiple myeloma cell adhesion to bone marrow stromal cells}

MM cell adhesion to BMSC through the interaction between VLA-4 and its corresponding ligand, VCAM-1, is among the predominant mechanisms for cell adhesionmediated drug resistance (CAM-DR) in $\mathrm{MM}^{22,29}$ while enhancing the production of IL- $6^{30}$ and RANKL, an critical osteoclastogenic factor in $\mathrm{MM}^{31}$ In addition to TAK1 activation in MM cells, TAK1 was found to be phosphorylated along with PIM2 upregulation in BMSC, when cocultured with MM cells (Figure 3A). Furthermore, VCAM-1 expression was substantially upregulated in BMSC after coculturing with MM cells, which was abolished by TAK1 inhibition with LLZ (Figure 3B). TNF- $\alpha$ is known as a potent inducer of VCAM-1 in BMSC through activation of the NF- $\kappa B$ signaling pathway. ${ }^{32,33}$ Treatment with LLZ as well as TAK1 knockdown by siRNA abrogated the upregulation of VCAM-1 expression in BMSC by TNF- $\alpha$ (Figures $3 \mathrm{~B}$ and $3 \mathrm{C}$ ). Treatment with TNF- $\alpha$ promptly phosphorylated TAK1 and degraded I $\mathrm{IB} \alpha$, and induced the phosphorylation of p38MAPK and ERK in BMSC (Figure 3D). However, TAK1 inhibition with LLZ as well as TAK1 knockdown abolished the degradation of I $\mathrm{B} \alpha$ and reduced the phosphorylation of p38MAPK and ERK in the presence of TNF- $\alpha$, indicating efficacious suppression 
A

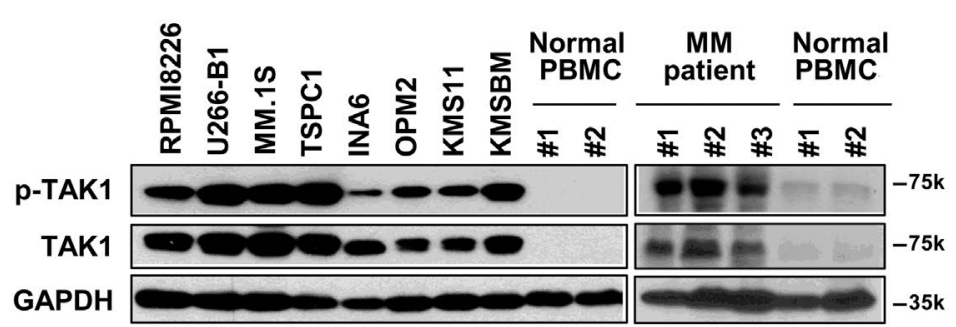

B $\quad \begin{array}{lllllllllllll}(\mu \mathrm{M}) & 0 & 1 & 5 & 0 & 1 & 5 & 0 & 1 & 5 & 0 & 1 & 5\end{array}$
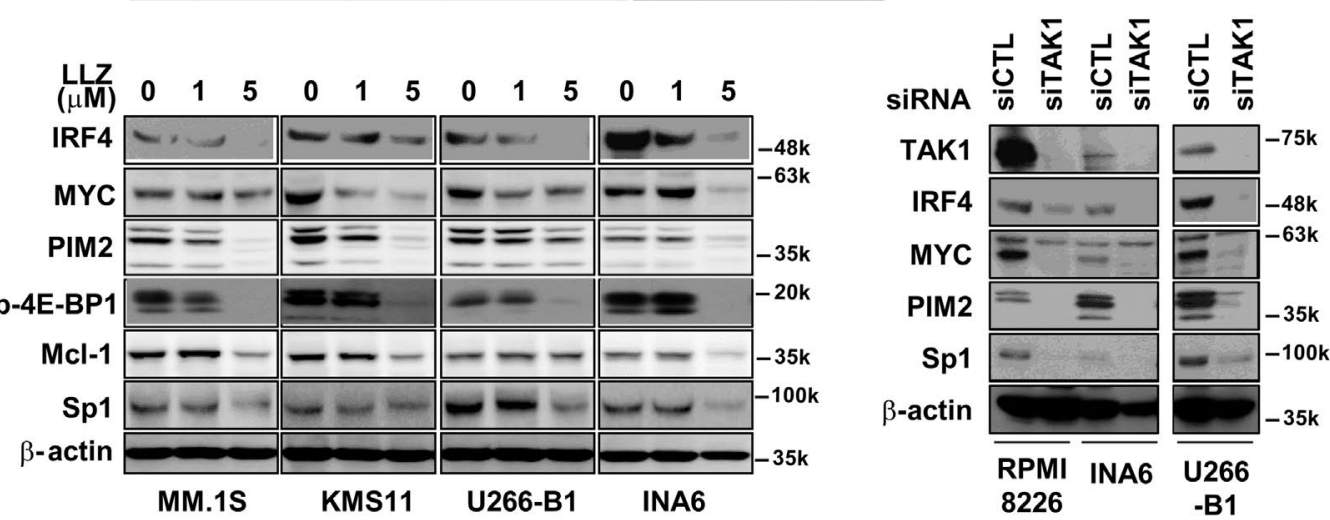

C
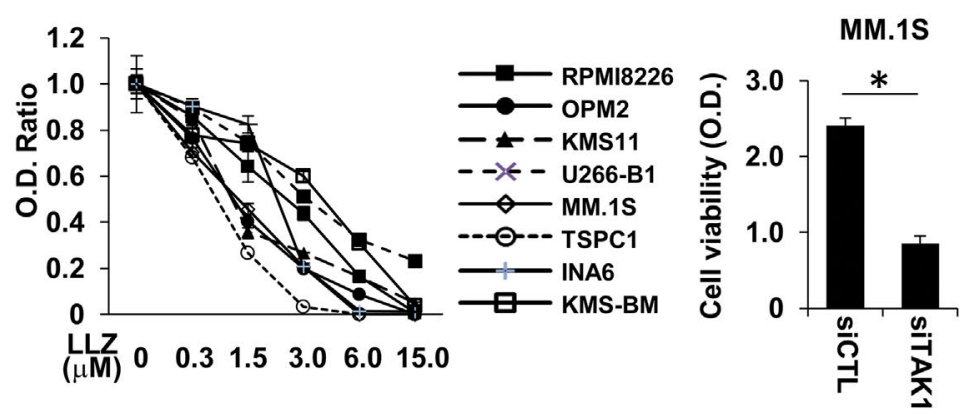

U266-B1

KMS11

D

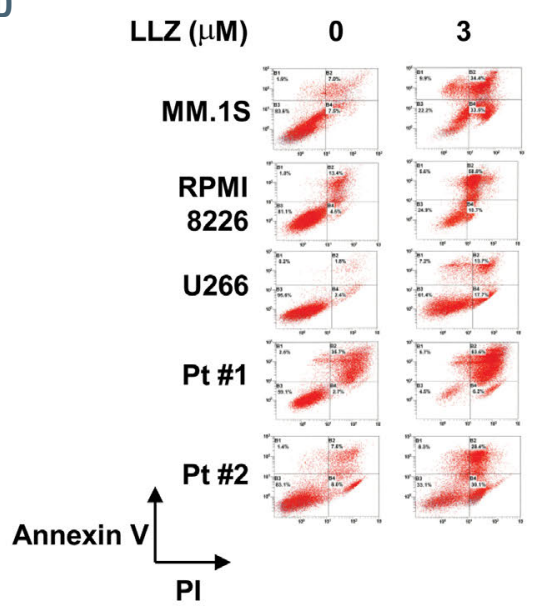

$\mathbf{E}$

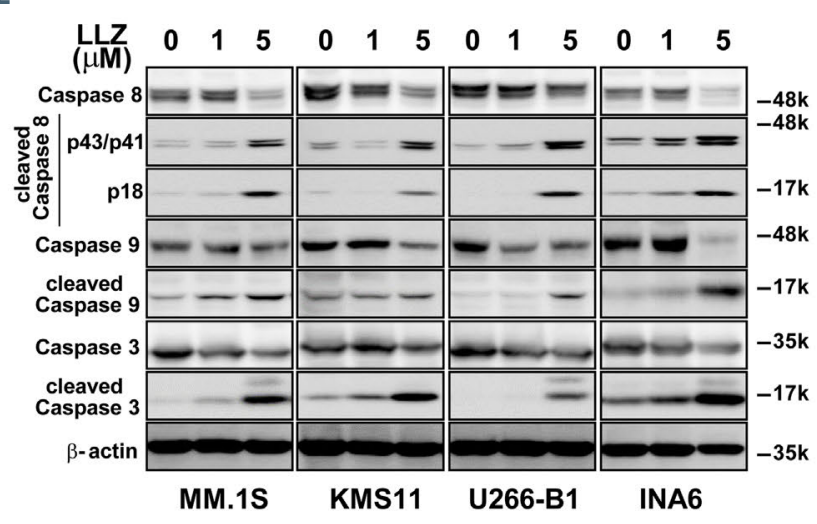

Figure 1. TAK1 is activated to mediate growth and survival of multiple myeloma cells. (A) TAK1 expression and phosphorylation in multiple myeloma (MM) cell lines TAK1 expression and phosphorylation were examined by western blotting analysis in various MM cell lines and primary MM cells as well as normal peripheral blood mononuclear cells (PBMC). Glyceraldehyde 3-phosphate dehydrogenase (GADPH) was used as a loading control. (B) The indicated MM cell lines were cultured in the absence or presence of LLZ at 1 or $5 u \mathrm{M}$ for 2 days (left). The cells were transduced with scrambled (siCTL) or human TAK1 small interfering RNA (siRNA) (siTAK1) and cultured for 13 hours (right). Cell lysates were then collected, and protein levels of the indicated molecules were analyzed by western blotting analysis. $\beta$-actin was used as a loading control. (C) MM cell lines as indicated were incubated in triplicate in the presence of the indicated concentrations of TAK1 inhibitor LLZ16402 (LLZ) for 48 hours (left). The indicated MM cell lines transduced with scrambled (siCTL) or human TAK1 siRNA (siTAK1) were cultured in triplicate for 24 hours (right). Cell viability was measured using a WST-8 assay. Data are expressed as means \pm standard deviation. (D) The indicated MM cell lines and CD138-positive cells isolated from MM patients (Pt \#1 and Pt \#2) were cultured in the presence or absence of LLZ at $3 \mu \mathrm{M}$ for 24 hours. The induction of apoptosis was analyzed using annexinV and propidium iodide (PI) dual staining. (E) The indicated MM cell lines were cultured alone or in the presence of LLZ at 1 or $5 \mu \mathrm{M}$ for 2 days, and cell lysates were then collected. Caspase-mediated apoptotic pathways were analyzed using western blotting analysis. $\beta$-actin was used as a loading control. 
A
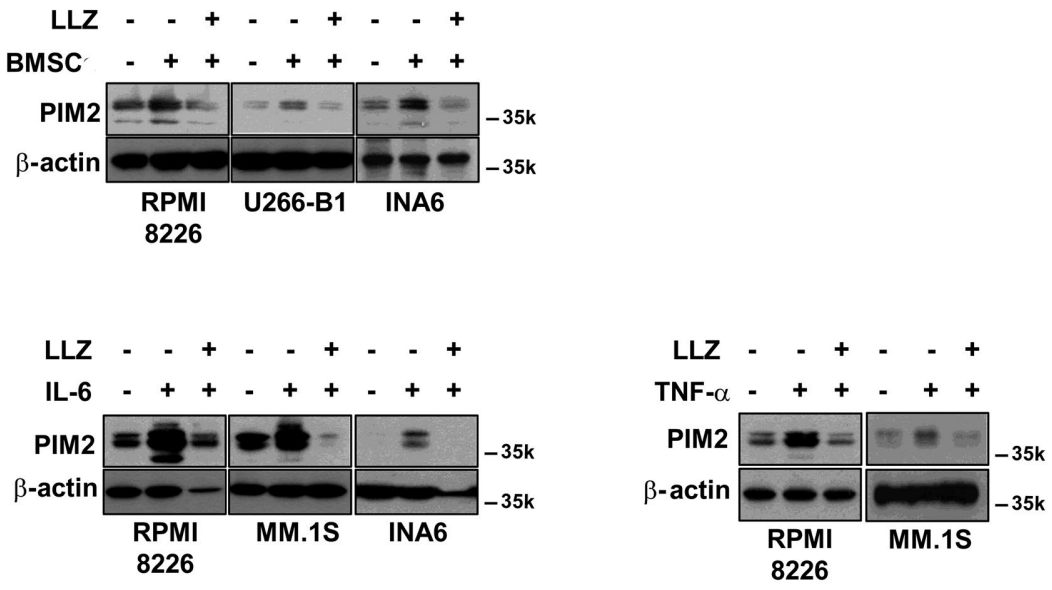

B

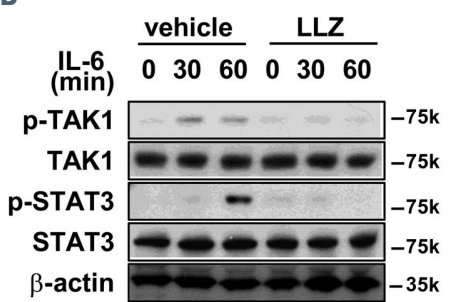

E

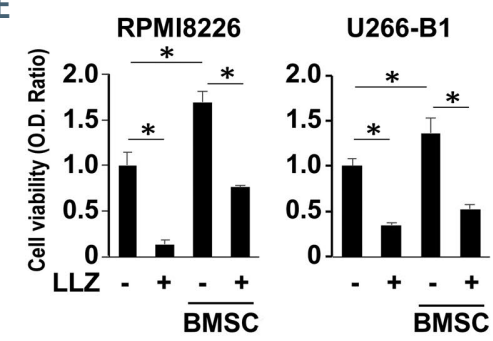

F

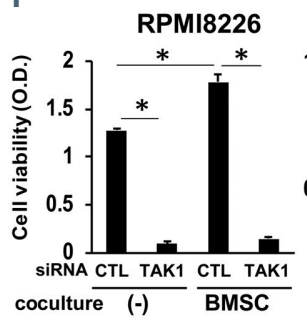

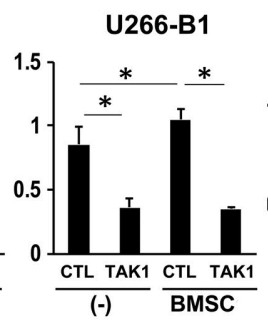

C

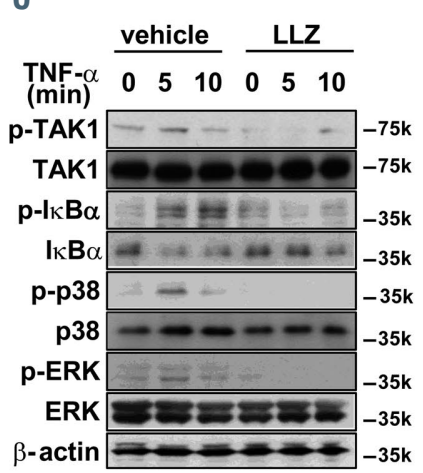

D

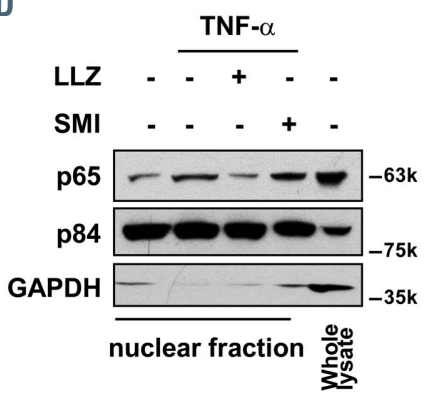

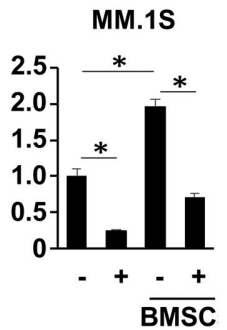

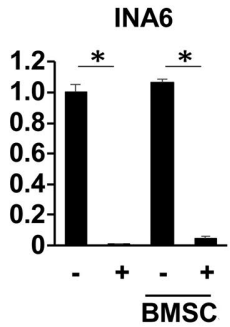

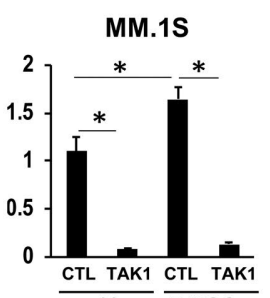

G

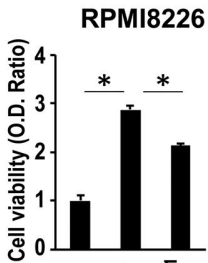

8.

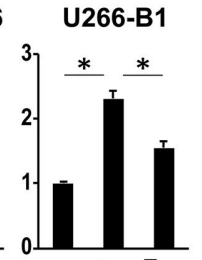

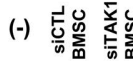

MM.1S

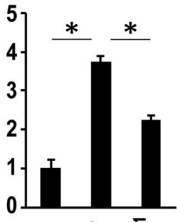

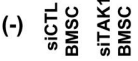

Figure 2. TAK1 inhibition abolishes IL-6- and TNF- $\alpha$-induced signaling in multiple myeloma cells. (A) Human bone marrow stromal cells (BMSC) were expanded in 24-well culture plates. The indicated multiple myeloma (MM) cell lines were cultured alone or cocultured with BMSC (upper), or cultured in the presence or absence of IL-6 (lower left) or TNF- $\alpha$ (lower right) for 24 hours (h), and cell lysates were then collected. PIM2 expression was analyzed using western blotting. $\beta$-actin was blotted as a loading control. (B, C) RPMI8226 cells were cultured in $\alpha$-MEM with $1 \%$ FBS for $12 \mathrm{~h}$ for serum starvation. The starved cells were then cultured in $\alpha$-MEM with $1 \%$ fetal bovine serum (FBS) with or without LLZ1640-2 (LLZ) at $3 \mu \mathrm{M}$. Three hours later, IL-6 (b) or TNF- $\alpha$ (c) at $10 \mathrm{ng} / \mathrm{mL}$ was added. After the indicated time periods, cell lysates were collected. The expression of phosphorylated TAK1 (p-TAK1), TAK1, phosphorylated STAT3 (p-STAT3), STAT3, phosphorylated IKB $\alpha$ (p-IKB $\alpha$ ), I $\mathrm{B} \alpha$, phosphorylated p38MAPK (p-p38), p38MAPK (p38), phosphorylated ERK (p-ERK), and ERK were detected using western blotting. $\beta$-actin was used as a loading control. (D) RPMI8226 cells after starvation were cultured in $\alpha$-MEM with $1 \%$ FBS with or without LLZ (5 $u$ M) or the PIM inhibitor SMI-16a (50 $u$ M). Three hours later, TNF- $\alpha$ at $10 \mathrm{ng} / \mathrm{mL}$ was added as indicated. After incubating for 15 minutes, whole lysates and nuclear fractions were extracted. The protein levels of p65 were analyzed using western blotting. Glyceraldehyde 3-phosphate dehydrogenase (GADPH) and p84 were used as protein loading controls for cytoplasmic and nuclear proteins, respectively. (E) The indicated MM cell lines were cultured alone or cocultured with human BMSC expanded in 24-well culture plates for 24 hours in the presence or absence of LLZ at $5 \mathrm{uM}$. MM cells were then collected, and their viability was analyzed using a WST8 assay. (F) The indicated MM cell lines were transduced with scrambled (siCTL) or TAK1 siRNA (siTAK1), cultured alone or cocultured with human BMSC expanded in 24-well culture plates for 24 hours. MM cells were then collected, and their viability was analyzed using a WST8 assay. (G) Indicated MM cells were cocultured for 24 hours with or without human BMSC which were transduced with scrambled (siCTL BMSC) or TAK1 siRNA (siTAK1 BMSC). MM cells were then collected, and their viability was analyzed using a WST8 assay. 
of TNF- $\alpha$ signaling in BMSC by TAK1 inhibition. VLA-4, a4 $\beta 1$ integrin, is constitutively overexpressed in $M M$ cells. We found that the TAK1 inhibition with LLZ was able to reduce the expression of $\beta 1$ integrin in $M M$ cells (Online Supplementary Figure S3), indicating the contribution of TAK1 to $\beta 1$ integrin expression in MM cells. Consistently, treatment with LLZ suppressed MM cell adhesion to BMSC (Figures 3E; Online Supplementary Figure S4), although LLZ did not impair the viability of BMSC (Online Supplementary Figure S5).

BMSC are regarded as a major source of IL-6, a growth and survival factor for MM cells, and the critical osteoclastogenic factor RANKL. Consistent with the previous observations ${ }^{30,31}$ cocultures with MM cells potently augmented IL-6 (Figures 3F) and RANKL (Figures 3G) mRNA expression in BMSC. However, treatment with LLZ suppressed the upregulation of these factors in BMSC in cocultures with MM cells. These data demonstrate that TAK1 inhibition is able to efficaciously suppress MM cell adhesion to BMSC and thereby abolish the upregulation of IL-6 and RANKL in BMSC, which may alleviate MM tumor progression in the bone marrow and bone destruction.

\section{TAK1 inhibition suppresses osteoclastogenesis enhanced by RANKL and multiple myeloma cells}

Consistent with previous observations, ${ }^{20,34}$ RANKL induced the phosphorylation of TAK1 in parallel with the degradation of IKB $\alpha$ and phosphorylation of p38MAPK and ERK (Figure 4A), and nuclear localization of the NF- $\mathrm{KB}$ subunit p65 (Figure 4B) in RAW264.7 preosteoclastic cells. However, treatment with LLZ abolished all of these RANKL-mediated changes, indicating critical involvement of TAK1 in RANKL-induced activation of the NF- $\mathrm{KB}$ and MAPK pathways. RANKL induced the expression of NFATc1 and c-fos, critical transcription factors for osteoclastogenesis (Figure 4C), and the formation of TRAP-positive multinucleated cells, namely OC, in RAW264.7 cells (Figures 4D); however, treatment with LLZ dose-dependently suppressed the RANKL-induced expression of NFATc1 and c-fos, and OC formation. TAK1 knockdown by siRNA also abolished the induction of NFATc1 and c-fos expression (Figure 4E) and osteoclastogenesis (Figure 4F) by RANKL. Furthermore, MM cells potently induced TRAP-positive multinucleated OC formation from bone marrow cells; however, treatment with LLZ suppressed OC formation (Figure 4G). These results demonstrate that TAK1 inhibition is able to suppress osteoclastogenesis enhanced by MM cells.

\section{TAK1 inhibition restores osteoblastogenesis suppressed by multiple myeloma cells as well as major inhibitors for osteoblastogenesis in multiple myeloma}

In contrast to the enhanced osteoclastogenesis, osteoblastogenesis or bone formation is suppressed in MM. Conditioned media (CM) from MM cell lines as well as inhibitory factors for osteoblastogenesis overproduced in $M M$, including IL-3, IL-7, TNF- $\alpha$, TGF- $\beta$, and activin $A,{ }^{8-12}$ induced the phosphorylation of TAK1 (Figure 5A) and suppressed mineralized nodule formation (Figure 5B) in MC3T3-E1 preosteoblastic cells. However, treatment with the TAK1 inhibitor LLZ restored mineralized nodule formation (Figure 5B). Osterix is an essential transcription factor for osteoblastogenesis, known as a downstream target of BMP-2. The upregulation of Osterix by BMP-2 was reduced in MC3T3-E1 cells in the presence of CM from MM cell lines or TNF- $\alpha$; however, treatment with LLZ restored the upregulation of Osterix by BMP-2 (Figure $5 \mathrm{C})$.

TGF- $\beta$ inhibits the terminal stage of OB differentiation or bone mineralization, whereas BMP-2 is a stimulator for osteoblastogenesis. ${ }^{11,35-38} \mathrm{We}$ and others demonstrated that TGF- $\beta$ plays a significant role in bone destruction in MM, and that the inhibition of the TGF- $\beta$ signaling restored bone formation in $\mathrm{MM}$ animal models. ${ }^{11,39-41}$ Treatment with TGF- $\beta$ induced the phosphorylation of Smad2 and Smad3 in MC3T3-E1 cells (Figure 5D). However, TAK1 inhibition with LLZ as well as TAK1 knockdown by siRNA abolished the phosphorylation of these factors. TGF- $\beta$ has been shown to counteract the BMP-2 signaling to suppress the terminal differentiation of $\mathrm{OB}$ in part through the upregulation of Smad6, an inhibitory regulator for BMP-2 signaling. ${ }^{42}$ Treatment with LLZ for 24 hours dose-dependently reduced Smad6 protein levels in MC3T3-E1 cells (Figure 5E, upper). Moreover, LLZ inhibited TGF- $\beta$-induced upregulation of Smad6 in MC3T3-E1 cells (Figure 5E, lower). In contrast, treatment with LLZ as well as TAK1 knockdown by siRNA enhanced the phosphorylation of Smad1/5 in MC3T3-E1 cells by BMP-2 (Figure 5F). These results collectively suggest that TAK1 inhibition may resume osteoblastogenesis suppressed in MM.

\section{TAK1 inhibition suppresses vascular endothelial growth factor secretion by multiple myeloma cells}

Angiogenesis also plays an important role in the pathogenesis and progression of MM. Vascular endothelial growth factor (VEGF) appears to be the most critical angiogenic factor in MM. ${ }^{43,44}$ VEGF has been demonstrated to be overproduced downstream of the signaling mediator ERK in MM cells. ${ }^{45}$ As expected, TAK1 inhibition with LLZ as well as TAK1 knockdown with siRNA substantially reduced VEGF production by MM cells (Online Supplementary Figure S6). These results suggested that TAK1 inhibition can impair angiogenesis in MM to retard MM progression.

\section{TAK1 inhibition suppresses multiple myeloma tumor progression and prevents bone destruction in vivo}

We next examined the in vivo effects of the TAK1 inhibitor LLZ using MM mouse models by intratibial inoculation of mouse 5TGM1 MM cells. Mice were treated with LLZ every other day for 2 weeks from day 6 , the day on which 5TGM1 MM cell-derived IgG2b levels started to increase in mouse sera. Vehicle-treated mice showed at day 21 large tumor masses around the tibiae where MM cells were inoculated (Figure 6A), and a progressive increase in serum IgG2b levels over time (Figure $6 \mathrm{~B}$ ). Bone destruction in the tibiae was observed at day 21 in plain $\mathrm{X}$-ray as well as $\mu$-computed tomography $(\mu$-CT) images (Figure 6C). Treatment with LLZ substantially suppressed tumor sizes (Figure 6A) with almost no increase in serum $\operatorname{IgG} 2 \mathrm{~b}$ (Figure $6 \mathrm{~B}$ ), and prevented bone destruction of the tibiae (Figure 6C). Cathepsin K-expressing OC increased in number on the surface of bone in 5TGM1-inoculated tibiae; however, treatment with LLZ reduced the OC numbers (Figure 6D). These results demonstrate that TAK1 inhibition is able to suppress MM tumor growth while preventing bone destruction in vivo.

In order to further clarify the effects of TAK1 inhibition 


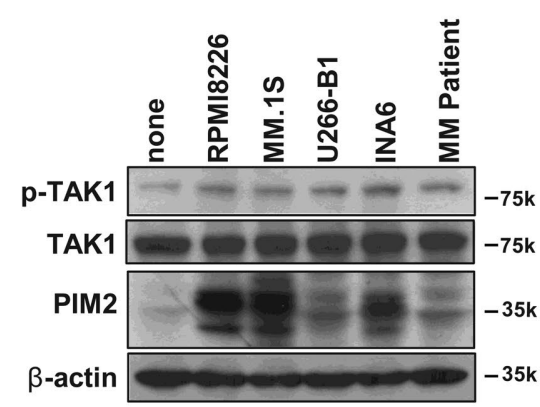

C

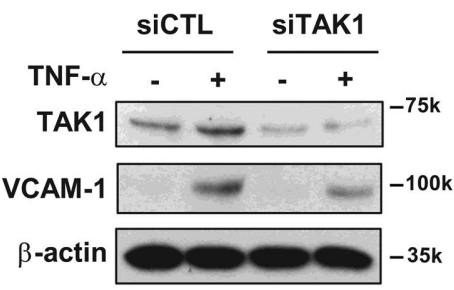

B

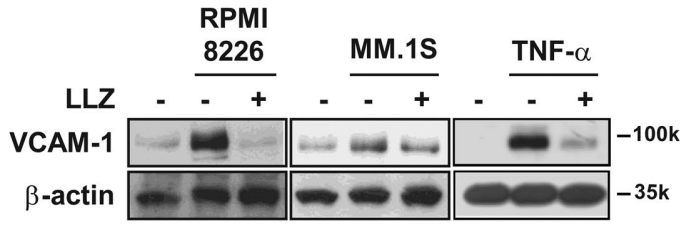

D
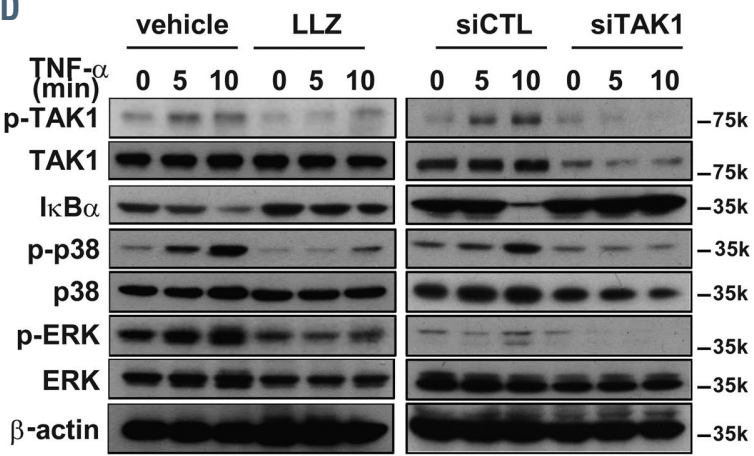

E
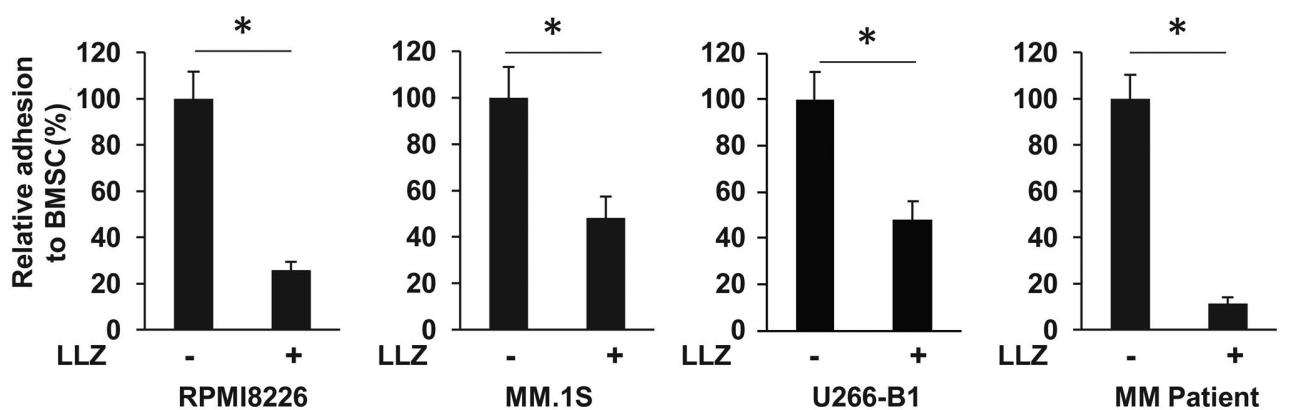

F
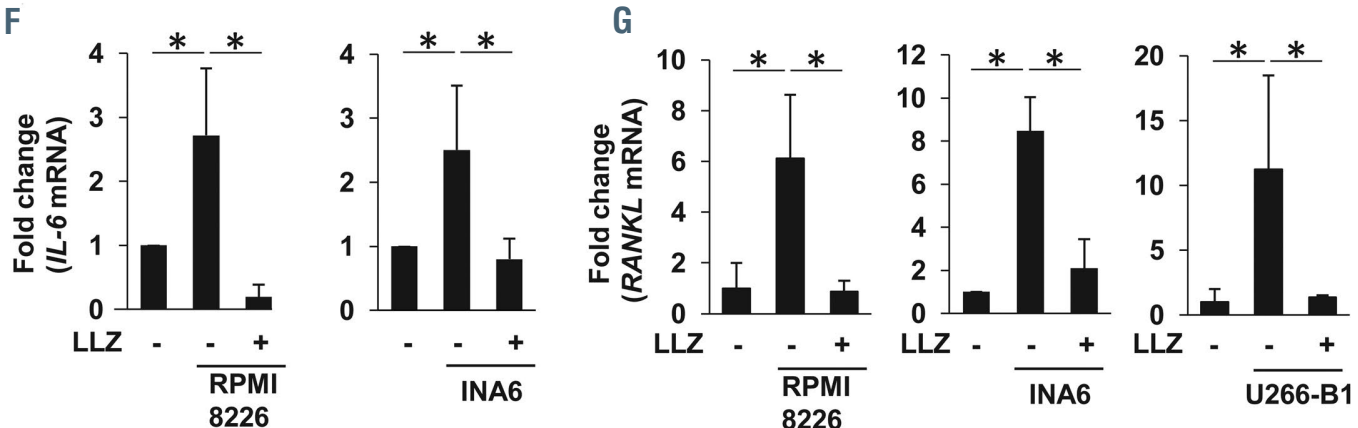

Figure 3. TAK1 inhibition suppresses VCAM-1 expression in bone marrow stromal cells and their adhesion to multiple myeloma cells. (A) Human bone marrow stromal cells (BMSC) were expanded in 6-well culture plates. The BMSC were cocultured with the indicated multiple myeloma (MM) cell lines for 24 hours. After washing out MM cells, cell lysates were collected from the BMSC. The indicated protein levels were examined using western blotting. (B) Human BMSC were cultured alone or cocultured with the indicated MM cell lines, or cultured with TNF- $\alpha$ at $10 \mathrm{ng} / \mathrm{mL}$ in the presence or absence of LLZ (5 $\mu$ M) for 2 days. Cell lysates were collected from BMSC, and VCAM-1 expression was analyzed using western blotting. (C) BMSC were transduced with scrambled (siCTL) or TAK1 small interfering RNA (siRNA) (siTAK1), and then cultured for 2 days with or without TNF- $\alpha$ at $10 \mathrm{ng} / \mathrm{mL}$. Cell lysates were collected, and VCAM-1 expression was analyzed using western blotting. (D) BMSC cells were starved in $\alpha$-MEM with $1 \%$ fetal bovine we periods. The indicated protein levels were analyzed using MM cells were then added in quadruplicate at $10^{5}$ cells/well, and incubated for 4 hours. By gentle pipetting, non-adherent MM cells were removed, and adherent MM cells were quantitated in a fluorescence multi-well plate reader. Data represent the means \pm standard deviation $(S D)(n=4)$. ${ }^{*} P<0.05$, by ANOVA. $(F, G)$ Human BMSC prepared in 6-well culture plates were cultured in triplicate alone or cocultured with MM cells as indicated in the presence or absence of LLZ ( $5 \mu \mathrm{M}$ ) for 1 day. After washing out MM cells, total RNA was isolated from the BMSC. IL-6 (f) and RANKL (g) mRNA expression in the BMSC was determined using quantitative reverse transcription polymerase chain reaction. Data represent the means $\pm S D(n=3)$. ${ }^{*} P<0.05$, by ANOVA. 
A

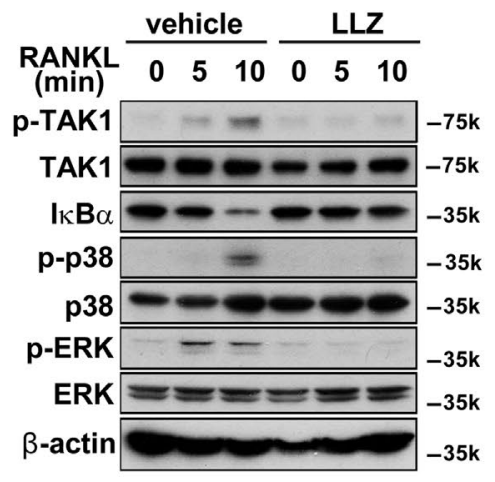

B

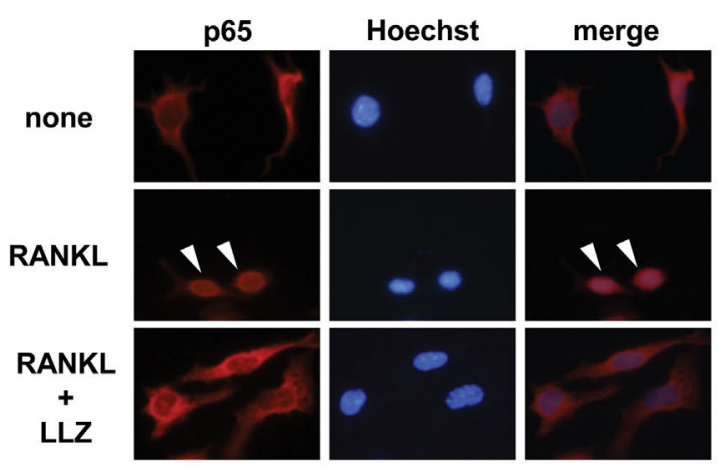

C

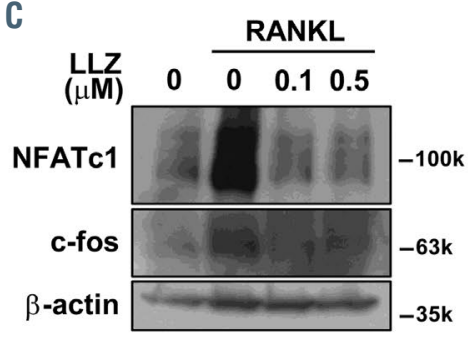

E

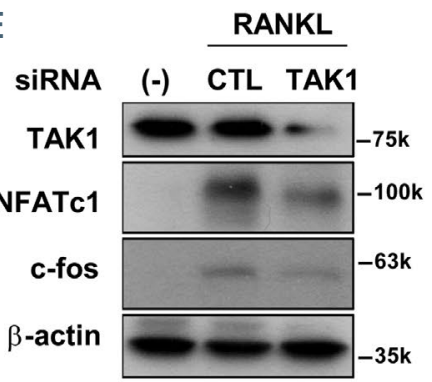

D

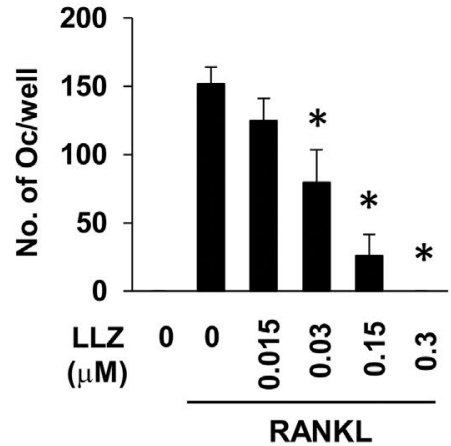

$\mathbf{F}$

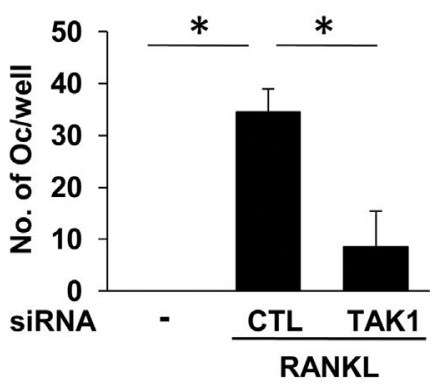

G
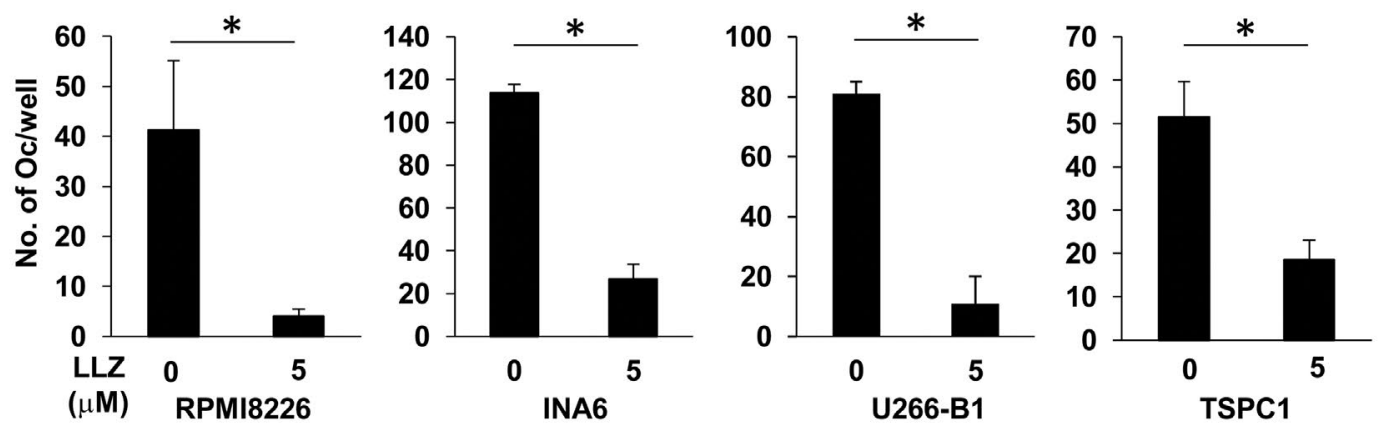

Figure 4. TAK1 inhibition suppresses osteoclastogenesis enhanced by RANKL and multiple myeloma cells. (A) RAW264.7 cells were starved in $\alpha$-MEM with $1 \%$ fetal bovine serum (FBS) for 12 hours, and then treated with or without LLZ at $1 \mu \mathrm{M}$ for 3 hours, followed by the addition of RANKL (20 ng/mL) for the indicated time peri-
ods. Phosphorylated TAK1 (p-TAK1), TAK1, IKB $\alpha$, phosphorylated p38MAPK (p-p38MAPK), p38MAPK, phosphorylated ERK (p-ERK), and ERK protein levels were anaods. Phosphorylated TAK1 ( $p$-TAK1), TAK1, IKB $\alpha$, phosphorylated p38MAPK (p-p38MAPK), p38MAPK, phosphorylated ERK (p-ERK), and ERK protein levels were ana-
lyzed using western blotting. $\beta$-actin was used as a loading control. (B) In order to observe p65 localization, the cells were fixed and stained with anti-p65 antibody (red), and their nuclei were stained with Hoechst 33342 (blue). (C) RAW264.7 cells were treated with or without RANKL at 25 ng/mL for 1 day. LLZ was added as indicated. Protein levels of NFATc1 and c-fos were determined using western blotting. (D) Primary mouse bone marrow cells were cultured in quadruplicate with or without RANKL $(25 \mathrm{ng} / \mathrm{mL})$ for 4 days. LLZ was added at the indicated concentrations. Then, the cells were fixed, and stained by TRAP. TRAP-positive multinucleated cells containing three or more nuclei were counted (left). Data are expressed as mean \pm standard deviation (SD). $* P<0.05$ (the difference from the results with RANKL without LZ). Microscopic images of TRAP staining in representative cultures are shown (right). Original magnification, $x 100$. (E) RAW264.7 cells were transfected with scrambi (STCT) or TAK1 (STAK1) Small interfering RNA, and then cultured with or without RANKL (25 ng/mL) for 24 hours. Cell lysates were then collected, with sAK1. NFATc1 and c( nuclei were counted. Data were expressed as mean \pm standard devaition (SD). ${ }^{*} P<0.05$. (G) Primary bone marrow cells derived from mice were cocultured in quadruplicate with MM cells as indicated for 4 days with or without LLZ. The cells were then fixed, and stained by TRAP. TRAP-positive multinucleated cells containing three or more nuclei were counted. Data were expressed as mean \pm SD. $* P<0.05$. 
A

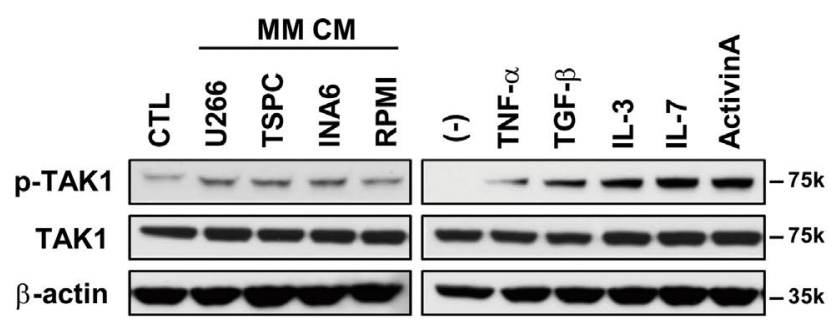

B

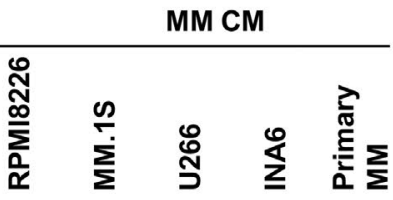

CTL

$(-)$

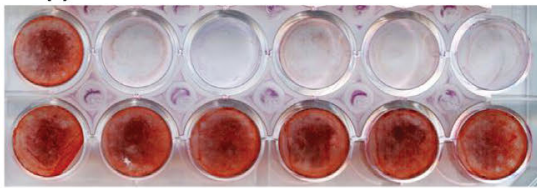

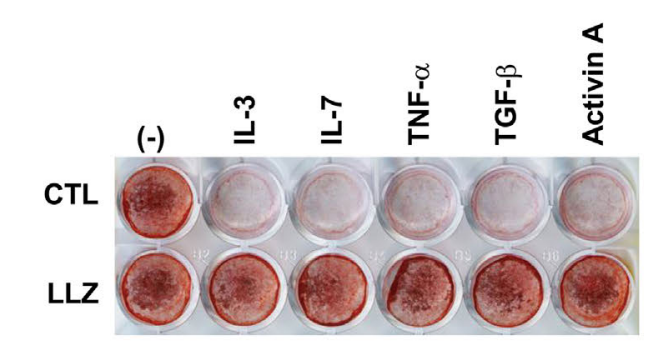

C
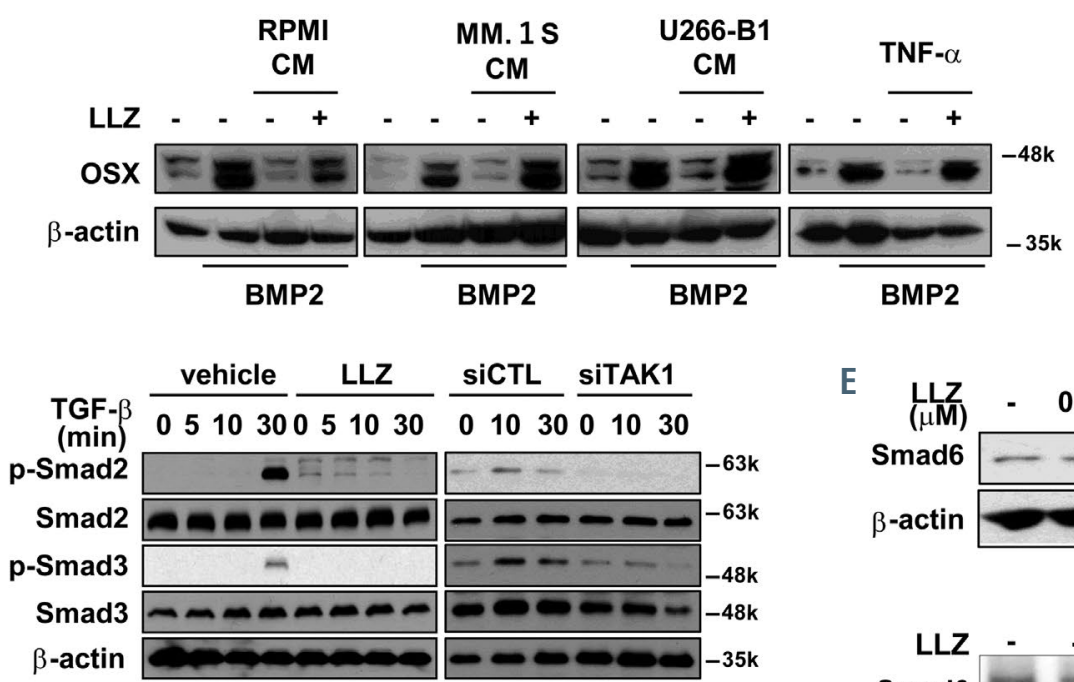

E
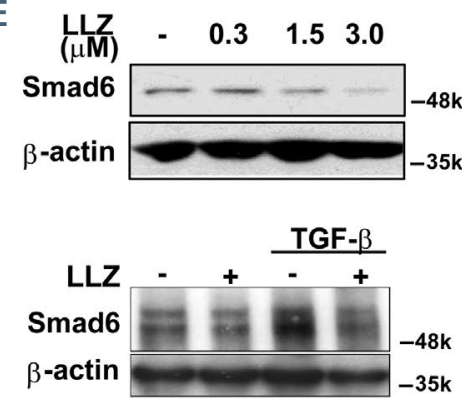

$F$

\begin{tabular}{|c|c|c|c|c|}
\hline \multirow[b]{2}{*}{$\begin{array}{c}\text { BMP2 } \\
(\min )\end{array}$} & \multirow{2}{*}{ vehicle } & LLZ & siCTL & SITAK1 \\
\hline & & $0 \quad 3060$ & 03060 & 03060 \\
\hline Smad1/5 & -- & -- & $=-1$ & --- \\
\hline Smad5 & $x=-5$ & $20-3$ & ---1 & --- \\
\hline$\beta$-actin & $-\infty$ & 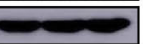 & -3 & 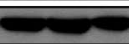 \\
\hline
\end{tabular}

Figure 5. TAK1 inhibition restores osteoblastogenesis suppressed by multiple myeloma cells as well as major inhibitors for osteoblastogenesis in multiple myeloma. (A) Conditioned media from the indicated multiple myeloma (MM) cell lines (MM CM) at $25 \%$, or cytokines including TNF- $\alpha$ (1 ng/ml), TGF- $\beta$ (10 ng/ml), lL-3 (10 $\mathrm{ng} / \mathrm{ml}), \mathrm{IL}-7(10 \mathrm{ng} / \mathrm{mL})$, or activinA $(10 \mathrm{ng} / \mathrm{mL})$ were added onto cultures with the MC3T3-E1 cells. After culturing for 24 hours, cell lysates were collected, and phosphorylated TAK1 (p-TAK1) and TAK1 levels were analyzed using western blotting. $B$-actin served as a loading control. (B) MC3T3-E1 cells were cultured in the presence or absence of LLZ $(0.3 \mu \mathrm{M})$ in osteogenic media with BMP-2 $(25 \mathrm{ng} / \mathrm{mL})$ in 24 -well culture plates. MM cells CM from the indicated cell lines and primary MM patient were added at $25 \%$. TNF- $\alpha(1 \mathrm{ng} / \mathrm{mL})$, TGF- $\beta(10 \mathrm{ng} / \mathrm{mL}), \mathrm{IL}-3(10 \mathrm{ng} / \mathrm{mL}), \mathrm{IL}-7(10 \mathrm{ng} / \mathrm{mL})$, or activinA $(10 \mathrm{ng} / \mathrm{mL})$ were added to the indicated wells. After culturing for 14 days, the cells were fixed and mineralized nodule formation was visualized using Alizarin red staining. (C) MC3T3-E1 cells were cultured for 4 days with MM CM $(25 \%)$ or TNF- $\alpha(1 \mathrm{ng} / \mathrm{mL})$ in the presence or absence of LLZ $(0.3 \mu \mathrm{M})$ in osteogenic media with BMP-2 $(25 \mathrm{ng} / \mathrm{mL})$. Then, cell lysates were collected and Osterix (OSX) expression was assayed using western blotting. (D) MC3T3-E1 cells were starved in $\alpha$-MEM with $1 \%$ FBS for 12 hours, and then treated with or without LLZ at $0.3 \mu \mathrm{M}$ for 3 hours, or transfected with scrambled (siCTL) or TAK1 (siTAK1) small interfering RNA. TGF- $\beta$ was added at $10 \mathrm{ng} / \mathrm{mL}$, and cell lysates were harvested after the indicated time periods. The expression of indicated proteins were analyzed using western blotting. (E) (Upper) MC3T3-E1 cells were treated with LLZ at the indicated concentrations for 12 hours. (Lower) MC3T3-E1 cells were treated with LIZ for 30 minutes prior to adding TGF- $\beta$ ( $10 \mathrm{ng} / \mathrm{mL}$ ) then cultured for 12 hours. The expression of Smad6 protein was analyzed using western blotting. (F) MC3T3-E1 cells were starved with or without LLZ at $0.3 \mu \mathrm{M}$ for 12 hours, or transfected with scrambled (SiCTL) or TAK1 (siTAK1) siRNA. BMP-2 was added at $25 \mathrm{ng} / \mathrm{mL}$, and cell lysates were harvested after the indicated time periods. The expression of indicated proteins were analyzed using western blotting. 
A

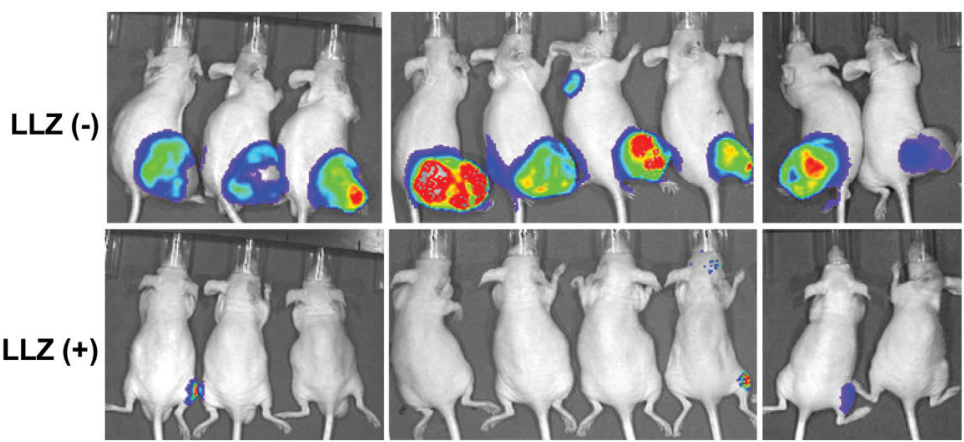

B

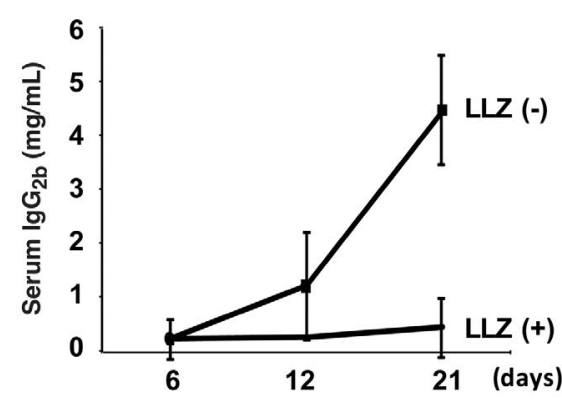

C

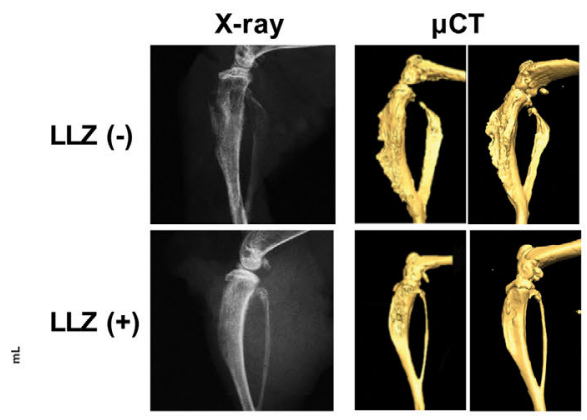

D

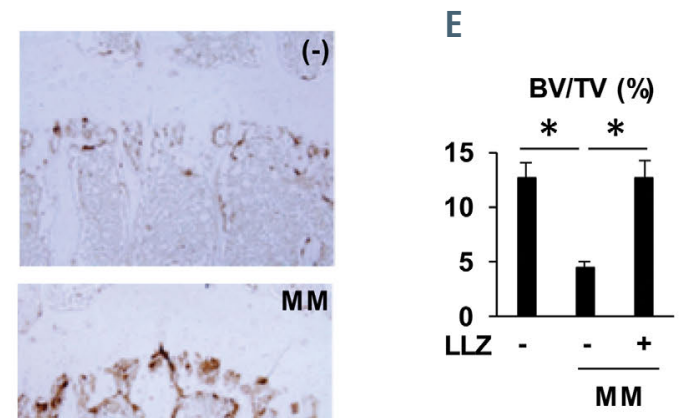

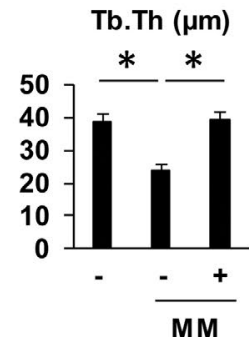

MM

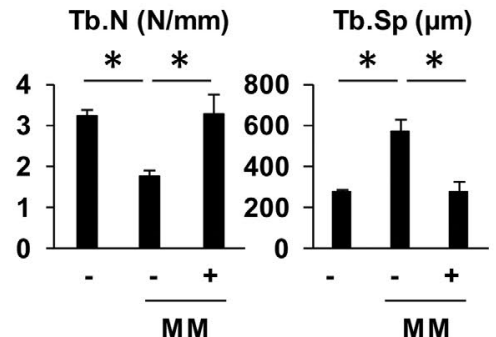

BFR/BS

$\left(\mathrm{mm}^{3} / \mathrm{mm}^{2} /\right.$ year $)$

N.Oc/BS (N/mm)

N.Ob/BS (N/mm)

OS/BS (\%)
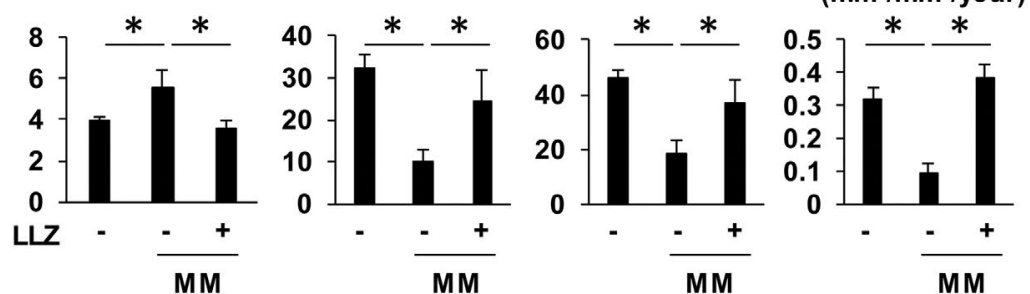

Figure 6. TAK1 inhibition suppresses multiple myeloma tumor growth and bone destruction in vivo. The mice with intra-tibial inoculation of luciferase-transfected $5 T$ TM 1 were treated with LLZ at $20 \mathrm{mg} / \mathrm{kg}$ or saline intraperitoneally every other day from days 6 to 21 . (A) Tumor sizes were assessed at day 21 via in vivo bioluminescence measurement using the IVIS Imaging System. For the luciferase detection imaging, $100 \mu \mathrm{L}$ of $15 \mathrm{mg} / \mathrm{mL}$ D-luciferin in phosphate-buffered saline was injected intraperitoneally before taking images. (B) Serum levels of mouse lgG ${ }_{2 b}$ were measured over time. (C) The tibiae with the tumor or control ones were extracted, and analyzed using soft X-ray (left) and $\mu$-computed tomography (right) imaging. (D) Immunohistochemical detection of cathepsin $\mathrm{K}$ in the tibia from a mouse inoculated with 5TGM1 multiple myeloma (MM) cells. (E) The tibiae were taken out at day 21 and analyzed histomorphometrically. The ratios of the bone volume to total volume (BV/TV), trabecular thickness (Tb.Th), trabecular numbers (Tb.N), trabecular separation (Tb.Sp), number of osteoclasts to bone surface (N.Oc/BS), number of osteoblasts to bone surface (N.Ob/BS) osteoid surface to bone surface (OS/BS), and bone formation rate to bone surface (BFR/BS) were analyzed using sections from three mice in each study arm. Data are expressed as mean \pm standard deviation. $* P<0.05$. 
on bone metabolism in MM, we histomorphometrically analyzed bone lesions in the mouse models. In vehicletreated mice, bone volume over total volume (BV/TV), trabecular thickness (Tb.Th), trabecular numbers (Tb.N), number of osteoblast surface over bone surface (N.Ob/BS), osteoid surface over bone surface (OS/BS), and bone formation rate over bone surface (BFR/BS) were decreased, whereas trabecular separation ( $\mathrm{Tb} . \mathrm{Sp}$ ) and the number of OC surface over bone surface (N.Oc/BS) were increased (Figure 6E). However, treatment with LLZ improved these changes in the 5TGM1-inoculated tibiae. These results demonstrate that the TAK1 inhibition not only suppresses osteoclastic bone destruction but also restores osteoid and bone formation in MM bone lesions. In order to further clarify the direct roles of TAK1 inhibition on pathological bone loss without tumor cells, we investigated the effects of LLZ on bone loss in ovariectomized (OVX) mice. In vehicle-treated OVX mice, bone loss was revealed in $\mu-\mathrm{CT}$; and BV/TV, Tb.Th and Tb.N were decreased, whereas Tb.Sp was increased in bone morphometric analysis (Online Supplementary Figure S7). However, treatment with LLZ was able to prevent OVXinduced pathological changes, suggesting a protective action of TAK1 inhibition on non-malignant bone loss.

\section{Discussion}

Although MM cells perturb bone metabolism with bone destruction, crosstalk between MM cells and the microenvironment in bone lesions leads to a progressive vicious cycle of tumor growth and bone destruction. The present study demonstrated that TAK1 plays a critical role in the vicious cycle, and suggested that TAK1 is a pivotal therapeutic target to disrupt the key signal transduction pathways responsible for tumor progression and bone destruction in MM. TAK1 activation appears to govern the expression of PIM2 in MM cells and osteoclastic as well as osteoblastic lineage cells; the TAK1-PIM2 signaling pathway is critical for MM tumor expansion and bone destruction. In addition to PIM2 upregulation, TAK1 activation induced Sp1 expression in MM cells. Sp1 is a ubiquitous zinc-finger transcription factor that binds guanine-cytosine-rich elements in the promoter region of its target genes and upregulates various important genes for cancer initiation and progression. ${ }^{46,47} \mathrm{Sp} 1$ is known to be constitutively overexpressed in many cancers and is associated with poor prognosis. ${ }^{46} \mathrm{Sp} 1$ expression and its DNA binding activity has been also demonstrated to be upregulated in MM cells. ${ }^{25}$ We and others reported that inhibition of Sp1 expression with Sp1 siRNA markedly induced apoptosis in MM cells, indicating that Sp1 as a novel therapeutic target for MM. ${ }^{25-27}$ Our results showed that TAK1 activation contributes to Sp1 over-expression in MM cells, and that TAK1 inhibition reduces Sp1 expression to impair MM cell growth and survival. TAK1 inhibition was found to reduce Sp1-mediated IRF4 expression in MM cells. As IMiD have been reported to downregulate IRF4 expression though degradation of IKZF1/3, 23,24 TAK1 inhibition may synergize the downregulation of IRF4 expression in combination with IMiD. TAK1 was also demonstrated to play a critical role in facilitating MM cell-BMSC adhesion via VLA-4VCAM-1 interaction. TAK1 inhibition reduced VCAM-1 expression in BMSC upregulated by MM cells or TNF- $\alpha$, and impaired MM cell adhesion to BMSC. MM cell-BMSC adhesion induced IL- 6 production and RANKL expression in BMSC in a manner dependent on TAK1 activation. Given that the adhesion of MM cells to BMSC via VLA-4VCAM-1 interaction confers CAM-DR in MM cells ${ }^{29,48,49}$ and osteoclastogenesis, ${ }^{50}$ these results suggested the therapeutic impact of TAK1 inhibition on CAM-DR as well as osteoclastogenesis induced by the MM-bone marrow interaction.

RANKL plays an important role in osteoclastogenesis enhanced in MM. As reported previously, ${ }^{34}$ RANKL induced the phosphorylation of TAK1 in RAW264.7 preosteoclastic cells in parallel with the degradation of I $\mathrm{B} \alpha$ and phosphorylation of p38MAPK and ERK (Figure 4A). However, TAK1 inhibition abolished these changes in the RANKL-mediated intracellular signaling and suppressed the formation of TRAP-positive OC from bone marrow cells upon treatment with exogenous RANKL (Figures 4D, F) as well as in cocultures with MM cells (Figure 4G). Together with suppression of the TAK1-dependent induction of RANKL expression in BMSC (Figure 3G), TAK1 inhibition can reduce osteoclastogenesis enhanced in MM through blockade of RANKLmediated signaling in osteoclastic lineage cells. As for osteoblastogenesis, major inhibitors for osteoblastogenesis in MM, including IL-3, IL-7, TNF- $\alpha$, TGF- $\beta$, and activinA, as well as MM cell CM-induced TAK1 phosphorylation while suppressing osteoblastogenesis in MC3T3-E1 preosteoblastic cells; however, TAK1 inhibition restored their osteoblastogenesis (Figures 5A,B). Taken together, these results underscored the value of TAK1 inhibition for preventing progression of bone destruction and restoring bone formation in MM. Finally, we validated the therapeutic effects of TAK1 inhibition in vivo. Treatment with LLZ markedly suppressed MM tumor growth and prevented bone destruction in mouse MM models with intra-tibial injection of 5TGM1 MM cells (Figure 6). Although various bone-modifying agents have been developed, bone loss still remains a serious unmet issue in patients with $\mathrm{MM}$; bone formation is hard to be restored in $\mathrm{MM}$ bone lesions by clinically available anti-resorptive agents, namely zoledronic acid and denosumab. In contrast to these agents, TAK1 inhibitors appear to be bone anabolic and anti-resorptive agents with tumor-suppressing activity, which may bring considerable benefits for patients with malignant diseases exhibiting bone loss, such as MM patients. Moreover, because TAK1 inhibition is a novel mechanism of action, combination with TAK1 inhibition can improve the therapeutic efficacy of currently available anti-cancer agents while preventing cancer-related and cancer treatment-induced bone loss. Therefore, TAK1 inhibition may be a promising therapeutic option with anti-tumor and bone modifying action, targeting the interaction between MM cells and their surrounding microenvironment. The present results warrant further study for development of novel TAK1 inhibitors useful for $\mathrm{MM}$ treatment. We are currently synthesizing novel compounds with better specificity for TAK1 with less toxicity. Further translational research will elucidate the dividends they may yield in improved clinical outcomes.

\section{Dislosures}

$M A$ received research funding from Chuagai Pharmaceutical, Sanofi K.K., Pfizer Seivaku K.K., Kyowa Hakko Kirin, MSD KK, Astellas Pharma, Takeda Pharmaceutical, Teijin Pharma and Ono Pharmaceutical, and honoraria from Daiichi Sankyo Company. The other authors have no conflicts of interest to declare. 


\section{Contributions}

JT and MA designed the research and conceived the project; $P C R$ was performed by JT, HT, AO and SS; flow cytometry by $A O, M H$ and $T H$; immunoblotting by JT, HT, MH, AO, AB, $T H, S N, M A, S S$ and $M I$; transfection by JT, HT, MH, AO and TH; and cell cultures by JT, HT, MH, AO, AB, TH, SN, MA, SS, MI, KS, MO, SF, KK and HM, JT, HT, MH, AO, TH, $S N, M H, I E, T H, T M$, and MA analyzed the data; JT and $M A$ wrote the manuscript.

\section{Funding}

This work was supported in part by JSPS KAKENHI Grant Numbers JP18K08329, JP16K11504, JP17KK0169, JP18H06294; and Aki Horinouchi Research Grant; Japan Leukemia Research Fund; Yasuda Memorial Medical Foundation; the Ichiro Kanehara Foundation; and the Research Clusters program of Tokushima University. The funders had no role in study design, data collection and analysis, decision to publish, or preparation of the manuscript.

\section{References}

1. Raje N, Roodman GD. Advances in the biology and treatment of bone disease in multiple myeloma. Clin Cancer Res. 2011; 17(6):1278-1286

2. Silbermann R, Roodman GD. Myeloma bone disease: pathophysiology and management. J Bone Oncol. 2013;2(2):59-69.

3. Tanaka Y, Abe M, Hiasa M, et al. Myeloma cell-osteoclast interaction enhances angiogenesis together with bone resorption: a role for vascular endothelial cell growth factor and osteopontin. Clin Cancer Res. 2007;13(3):816-823.

4. Cackowski FC, Anderson JL, Patrene KD, et al. Osteoclasts are important for bone angiogenesis. Blood. 2010;115(1):140-149.

5. Asano J, Nakano A, Oda A, et al. The serine/threonine kinase Pim-2 is a novel antiapoptotic mediator in myeloma cells. Leukemia. 2011;25(7):1182-1188.

6. Lu J, Zavorotinskaya T, Dai Y, et al. Pim2 is required for maintaining multiple myeloma cell growth through modulating TSC2 phosphorylation. Blood. 2013;122(9):16101620.

7. Johrer K, Obkircher M, Neureiter D, et al. Antimyeloma activity of the sesquiterpene lactone cnicin: impact on Pim-2 kinase as a novel therapeutic target. J Mol Med (Berl). 2012;90(6):681-693.

8. Ehrlich LA, Chung HY, Ghobrial I, et al. IL3 is a potential inhibitor of osteoblast differentiation in multiple myeloma. Blood. 2005;106(4):1407-1414.

9. Giuliani N, Colla S, Morandi F, et al. Myeloma cells block RUNX2/CBFA1 activity in human bone marrow osteoblast progenitors and inhibit osteoblast formation and differentiation. Blood. 2005; 106(7):2472-2483.

10. D'Souza S, del Prete D, Jin S, et al. Gfi1 expressed in bone marrow stromal cells is a novel osteoblast suppressor in patients with multiple myeloma bone disease. Blood. 2011;118(26):6871-6880.

11. Takeuchi K, Abe M, Hiasa M, et al. TgfBeta inhibition restores terminal osteoblast differentiation to suppress myeloma growth. PloS One. 2010;5(3):e9870.

12. Vallet S, Mukherjee S, Vaghela N, et al. Activin A promotes multiple myelomainduced osteolysis and is a promising target for myeloma bone disease. Proc Natl Acad Sci U S A. 2010;107(11):5124-5129.

13. Hiasa M, Teramachi J, Oda A, et al. Pim-2 kinase is an important target of treatment for tumor progression and bone loss in myeloma. Leukemia. 2015;29(1):207-217.

14. Teramachi J, Hiasa M, Oda A, et al. Pim-2 is a critical target for treatment of osteoclastogenesis enhanced in myeloma. $\mathrm{Br} \mathrm{J}$ Haematol. 2018;180(4):581-585.

15. Mihaly SR, Ninomiya-Tsuji J, Morioka S. TAK1 control of cell death. Cell Death
Differ. 2014;21(11):1667-1676.

16. Sakurai H. Targeting of TAK1 in inflammatory disorders and cancer. Trends Pharmacol Sci. 2012;33(10):522-530.

17. Buglio D, Palakurthi S, Byth $K$, et al. Essential role of TAK1 in regulating mantle cell lymphoma survival. Blood. 2012 120(2):347-355

18. Safina A, Ren MQ, Vandette E, Bakin AV. TAK1 is required for TGF-beta 1-mediated regulation of matrix metalloproteinase- 9 and metastasis. Oncogene. 2008; 27(9):1198-1207.

19. Singh A, Sweeney MF, Yu M, et al. TAK1 inhibition promotes apoptosis in KRAS dependent colon cancers. Cell. 2012 148(4):639-650

20. Tenshin H, Teramachi J, Oda A, et al. TAK1 inhibition subverts the osteoclastogenic action of TRAIL while potentiating its antimyeloma effects. Blood Adv. 2017 1(24):2124-2137.

21. Wang LH, Yang XY, Zhang X, Farrar WL. Inhibition of adhesive interaction between multiple myeloma and bone marrow stromal cells by PPARgamma cross talk with NF-kappaB and C/EBP. Blood. 2007; 110(13):4373-4384

22. Abe M, Hiura K, Ozaki S, Kido S, Matsumoto T. Vicious cycle between myeloma cell binding to bone marrow stromal cells via VLA-4-VCAM-1 adhesion and macrophage inflammatory protein-1alpha and MIP-1beta production. J Bone Miner Metab. 2009;27(1):16-23

23. Zhu YX, Braggio E, Shi CX, et al. Identification of cereblon-binding proteins and relationship with response and surviva after IMiDs in multiple myeloma. Blood. 2014;124(4):536-545

24. Tang S, Ma D, Cheng B, et al. Crucial role of HO-1/IRF4-dependent apoptosis induced by panobinostat and lenalidomide in multiple myeloma. Exp Cell Res. 2018;363(2):196-207.

25. Fulciniti M, Amin S, Nanjappa P, et al. Significant biological role of sp1 transactivation in multiple myeloma. Clin Cancer Res. 2011;17(20):6500-6509.

26. Bat-Erdene A, Miki H, Oda A, et al Synergistic targeting of Sp1, a critical transcription factor for myeloma cell growth and survival, by panobinostat and proteasome inhibitors. Oncotarget. 2016;7(48):79064-79075

27. Kikuchi J, Wada T, Shimizu R, et al Histone deacetylases are critical targets of bortezomib-induced cytotoxicity in multiple myeloma. Blood. 2010;116(3):406-417.

28. Yasui H, Hideshima T, Richardson PG, Anderson KC. Novel therapeutic strategies targeting growth factor signalling cascades in multiple myeloma. Br J Haematol. 2006; 132(4):385-397.

29. Damiano JS, Cress AE, Hazlehurst LA, Shtil AA, Dalton WS. Cell adhesion mediated drug resistance (CAM-DR): role of integrins and resistance to apoptosis in human myeloma cell lines. Blood. 1999;93(5):1658 1667

30. Chauhan D, Uchiyama H, Akbarali Y, et al. Multiple myeloma cell adhesion-induced interleukin-6 expression in bone marrow stromal cells involves activation of NFkappa B. Blood. 1996;87(3):1104-1112.

31. Giuliani N, Colla S, Morandi F, Rizzoli V. The RANK/RANK ligand system is involved in interleukin- 6 and interleukin-11 up-regulation by human myeloma cells in the bone marrow microenvironment. Haematologica. 2004;89(9):1118-1123.

32. Hiruma Y, Honjo T, Jelinek DF, et al. Increased signaling through $\mathrm{p} 62$ in the marrow microenvironment increases myeloma cell growth and osteoclast formation. Blood. 2009;113(20):4894-4902.

33. Teramachi J, Silbermann R, Yang P, et al. Blocking the ZZ domain of sequestosome1/p62 suppresses myeloma growth and osteoclast formation in vitro and induces dramatic bone formation in myeloma-bearing bones in vivo. Leukemia. 2016;30(2):390-398

34. Mizukami J, Takaesu G, Akatsuka H, et al. Receptor activator of NF-kappaB ligand (RANKL) activates TAK1 mitogen-activated protein kinase kinase kinase through a signaling complex containing RANK, TAB2, and TRAF6. Mol Cell Biol. 2002;22(4):992-1000.

35. Spinella-Jaegle S, Roman-Roman S, Faucheu C, et al. Opposite effects of bone morphogenetic protein-2 and transforming growth factor-beta1 on osteoblast differentiation. Bone. 2001;29(4):323-330.

36. Maeda S, Hayashi M, Komiya S, Imamura T, Miyazono K. Endogenous TGF-beta signaling suppresses maturation of osteoblastic mesenchymal cells. EMBO J. 2004;23(3):552-563.

37. Miyazono K. TGF-beta signaling by Smad proteins. Cytokine Growth Factor Rev. 2000;11(1-2):15-22

38. Alliston T, Choy L, Ducy P, Karsenty G, Derynck R. TGF-beta-induced repression of CBFA 1 by Smad3 decreases cbfa1 and osteocalcin expression and inhibits osteoblast differentiation. EMBO J. 2001;20(9):2254-2272.

39. Matsumoto T, Abe M. TGF-beta-related mechanisms of bone destruction in multiple myeloma. Bone. 2011;48(1):129-134.

40. Nyman JS, Merkel AR, Uppuganti S, et al Combined treatment with a transforming growth factor beta inhibitor (1D11) and bortezomib improves bone architecture in a mouse model of myeloma-induced bone disease. Bone. 2016;91:81-91.

41. Lu A, Pallero MA, Lei W, et al. Inhibition of transforming growth factor-beta activation diminishes tumor progression and osteolytic bone disease in mouse models of multi- 
ple myeloma. Am J Pathol. 2016;186(3): 678-690.

42. Fujii M, Takeda K, Imamura T, et al. Roles of bone morphogenetic protein type I receptors and Smad proteins in osteoblast and chondroblast differentiation. Mol Biol Cell. 1999;10(11):3801-3813.

43. Podar K, Tai YT, Davies FE, et al. Vascular endothelial growth factor triggers signaling cascades mediating multiple myeloma cell growth and migration. Blood. 2001; 98(2):428-435.

44. Bellamy WT. Expression of vascular endothelial growth factor and its receptors in multiple myeloma and other hematopoietic malignancies. Semin Oncol. 2001; 28(6):551-559.
45. Giuliani N, Lunghi P, Morandi F, et al Downmodulation of ERK protein kinase activity inhibits VEGF secretion by human myeloma cells and myeloma-induced angiogenesis. Leukemia. 2004;18(3):628635.

46. Beishline K, Azizkhan-Clifford J. Sp1 and the 'hallmarks of cancer'. FEBS J. 2015 28282):224-258

47. Tornin J, Martinez-Cruzado L, Santos L, et al. Inhibition of SP1 by the mithramycin analog EC-8042 efficiently targets tumor initiating cells in sarcoma. Oncotarget. 2016;7(21):30935-30950.

48. Landowski TH, Olashaw NE, Agrawal D, Dalton WS. Cell adhesion-mediated drug resistance (CAM-DR) is associated with activation of NF-kappa B (RelB/p50) in myeloma cells. Oncogene. 2003;22(16): 2417-2421.

49. Hazlehurst LA, Damiano JS, Buyuksal I, Pledger WJ, Dalton WS. Adhesion to fibronectin via beta1 integrins regulates p27kip1 levels and contributes to cell adhesion mediated drug resistance (CAM-DR) Oncogene. 2000;19(38):4319-4327.

50. Michigami T, Shimizu N, Williams PJ, et al. Cell-cell contact between marrow stromal cells and myeloma cells via VCAM-1 and alpha(4)beta(1)-integrin enhances production of osteoclast-stimulating activity. Blood. 2000;96(5):1953-1960 